\title{
Modelos não lineares ajustados à produção acumulada de biogás provenientes de camas sobrepostas de suínos
}

Sílvio de Castro Silveira ${ }^{1}$

Joel Augusto Muniz²

Francine Aparecida Sousa ${ }^{3}$

Alessandro Torres Campos ${ }^{4}$

\section{Resumo}

Enfrentam-se atualmente diversos problemas com a escassez de chuvas, um deles é a baixa produção de energia hidrelétrica e a consequente elevação das tarifas de energia. Os criadores são diretamente prejudicados, visto que essa variação incide em elevação nos custos de produção. Uma saída para os criadores é aproveitar os resíduos provenientes das atividades de plantio e criação para a produção do biogás por meio de biodigestores e geração de energia. Além do benefício do biogás e da energia, a utilização dos resíduos nos biodigestores promove o saneamento rural e produz biofertilizante de alta qualidade. Este trabalho trata-se de um estudo de regressão com os modelos não lineares sigmoide de Boltzmann, Gompertz e Logístico utilizando-se de dados de um projeto desenvolvido por Sousa (2014), com o objetivo de verificar qual modelo de regressão não linear melhor se ajusta à produção acumulada de biogás de biodigestores, modelo batelada, com material diluído de cama de suíno preparada com maravalha e bagaço de cana e somente com maravalha. Verificou-se que o modelo não linear sigmoide de Boltzmann é superior aos demais modelos e por consequência mais eficiente para o dimensionamento da produção de biogás e energia elétrica.

Palavras-chave: Energia elétrica. Biodigestores. Sigmoide de Boltzmann.

\section{Introdução}

Em períodos de escassez de chuvas, tem-se como consequência, entre outras, a redução na produção de energia hidrelétrica. Para suprir essa redução de energia, aumenta-se a geração desta por meio de termoelétricas, o que acarreta um aumento nas tarifas de energia, além de promover aumento da poluição, pois para o seu funcionamento depende-se da queima de gás, biomassa, óleo diesel e carvão mineral.

Para os criadores serem menos prejudicados, uma alternativa é a produção de energia elétrica utilizando biogás proveniente de biodigestores. Os biodigestores são equipamentos utilizados para promover a biodigestão anaeróbia, isto é, promover a reação entre a matéria orgânica (dejetos de

1 Universidade Federal de Lavras/UFLA, doutor em Estatística e Experimentação Agropecuária. silviodecs@gmail.com. Departamento de Estatística, caixa postal 37, Campus Universitário da UFLA, Lavras, Minas Gerais, CEP 37200-000.

2 Universidade Federal de Lavras/UFLA, professor titular. joamuniz@des.ufla.br.

3 Prefeitura Municipal de Aracruz/Secretaria Municipal de Agricultura, gerente do Serviço de Inspeção Municipal. fsousa@aracruz.es.gov.br.

4 Universidade Federal de Lavras/UFLA, professor associado. campos@deg.ufla.br. 
suínos, bovinos e aves entre outros) e microrganismos anaeróbios, resultando na produção de biogás e biofertilizante.

O biogás é constituído por uma mistura gasosa combustível, composta principalmente por dois gases, o metano $\left(\mathrm{CH}_{4}\right)$, que representa normalmente de 60 a 70\% da mistura, e o dióxido de carbono $\left(\mathrm{CO}_{2}\right)$, que representa de 30 a 40\% (COLDEBELLA et al., 2008).

Estudos têm demonstrado que a produção de energia elétrica por meio do biogás é economicamente viável, principalmente quando esta é utilizada para substituir ou mesmo para reduzir a aquisição de energia elétrica da concessionária (AVACI et al., 2013; MARTINS; OLIVEIRA, 2011).

Além de produzir energia com menor custo, a produção do biogás contribui para o saneamento rural. De acordo com Queiroz (2003), os resíduos, se despejados de forma inadequada, resultam em uma das principais formas de contaminação do meio ambiente, além de propagarem doenças infecciosas. Nishimura (2009) destacou ainda a contaminação dos solos e das águas subterrâneas e de superfície.

Os benefícios da produção de biogás também são destacados por Avaci et al. (2013), que mostraram que uma parte significativa dos custos de produção de energia pode ser compensada com a produção de biofertilizante para a produção de grãos e pastagens, o que torna a produção do biogás ainda mais competitiva em tempos de alta de insumos agrícolas. Segundo Luna et al. (2009), o biofertilizante tem sido utilizado na agricultura em substituição aos fertilizantes minerais.

É importante estabelecer a relação entre a quantidade de resíduos e o volume de biogás produzido da maneira mais verossímil possível, pois ela servirá de base para o correto dimensionamento de todo o sistema de geração de energia.

Trabalhos anteriores utilizaram modelos não lineares para descrever essa relação em diferentes situações, Queiroz (2003) utilizou modelos não lineares para ajustar o volume de biogás produzido com dejetos bovinos. Florentino, Biscaro e Passos (2010) estudaram diversos modelos de regressão não lineares na avaliação da atividade metanogênica específica de lodos anaeróbios. Lopes (2007) também utilizou modelos não lineares para avaliar diferentes composições de material de abastecimento de uma rede de biodigestores.

O uso de modelos não lineares para ajuste de curvas vem sendo largamente ampliado, pois esses modelos podem ser utilizados nas mais variadas áreas do conhecimento, devido ao fato de apresentarem boa qualidade de ajuste com uma quantidade pequena de parâmetros com interpretações práticas. Corrêa Filho et al. (2015) utilizaram modelos não lineares na descrição da cinética de secagem do figo em diferentes temperaturas. Carneiro et al. (2014) avaliaram modelos de crescimento de bovinos da raça tabapuã criados no nordeste do Brasil e obtiveram resultados satisfatórios.

Diversos trabalhos de modelos não lineares também avaliaram a dependência residual (PRADO et al., 2013a; SOUZA et al.,2014) e mostraram que modelos não lineares com estrutura de erros autorregressivos apresentam melhor qualidade de ajuste e estimam os parâmetros dos modelos mais próximos da realidade. Prado et al. (2013a) ainda afirmaram que, em casos de séries cronológicas de dados, a não consideração da correlação residual pode levar à obtenção de estimativas viesadas e à subestimação das variâncias dos parâmetros.

A modelagem da produção de biogás, por meio de modelos de regressão não lineares, com o uso de parâmetros autorregressivos, ainda não foi verificada na literatura para as condições deste trabalho.

Pela definição, modelo não linear é aquele em que pelo menos uma das derivadas do modelo em relação aos parâmetros depende dos parâmetros. Logo, não é possível isolar os parâmetros e 
obter uma solução explícita para o sistema. Para resolvê-lo, recorre-se ao uso de métodos numéricos iterativos (DRAPER; SMITH, 1998).

O presente trabalho tem por objetivo modelar a produção de biogás acumulada, utilizando-se de modelos de regressão não linear no processo de biodigestão anaeróbia, a partir de cama sobreposta de suínos diluída em água e selecionar o modelo de regressão que melhor se ajusta aos dados.

\section{Material e métodos}

Os dados são provenientes de um projeto desenvolvido por Sousa (2014) no Laboratório de Construções Rurais, Ambiência e Tratamento de Resíduos do Departamento de Engenharia da Universidade Federal de Lavras (DEG/UFLA), no município de Lavras (MG).

Foram utilizados doze protótipos de biodigestores laboratoriais, fabricados em recipientes plásticos retangulares, de base 23,5 x 33,0 cm e altura de 37,6 cm, com um volume total de 22,3 litros, de acordo com a fabricante Emplas, sendo o volume utilizado de 20 litros. Esses biodigestores são conectados por um tubo de plástico cristal a gasômetros independentes confeccionados em tubos de policloreto de vinila (PVC) de $25 \mathrm{~cm}$ de diâmetro, encaixados em tubos de PVC de $20 \mathrm{~cm}$ de diâmetro, com capacidade útil de armazenamento de 15 litros cada, com finalidade de armazenar e mensurar o biogás produzido conforme ilustrado na Figura 1.

Figura 1. Protótipo de biodigestor modelo batelada utilizado no processo de biodigestão anaeróbia e produção de biogás.

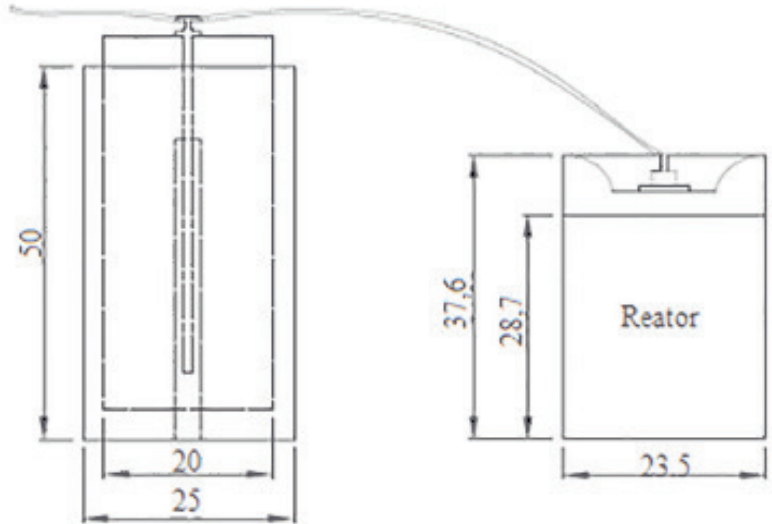

\section{Escala 1:10}

Unidade: $\mathrm{cm}$

Fonte: Sousa (2014).

Os ensaios conduzidos nesses biodigestores foram de batelada com abastecimento único por período e coleta diária de gás. Os materiais de abastecimento foram dejetos de suínos compostos com as seguintes camas: cama de maravalha + bagaço de cana $(M+B)$ e cama de maravalha (MAR), ambas provenientes de instalação em fase de terminação. Para uso no biodigestor, a cama foi diluída na proporção de 4 kg de dejetos, 2 kg de inóculo de dejeto de suíno e 14 kg de água com tempo de retenção hidráulica de 60 dias. 
Foi quantificada a produção de biogás a fim de verificar o potencial energético da cama sobreposta de suínos. Tal quantificação foi feita pela medição do deslocamento vertical do gasômetro. 0 volume do biogás foi determinado pela multiplicação da altura de deslocamento do gasômetro pela área de sua secção transversal interna. Após cada leitura, os gasômetros foram esvaziados até atingirem o zero da escala. A correção do volume de biogás para as condições de 101325 Pa e 293,15 K foi efetuada com base no trabalho de Caetano (1985), utilizando a equação 1:

$$
\left(V_{0} \times P_{0}\right) / T_{0}=\left(V_{1} \times P_{1}\right) / T_{1}
$$

em que:

$\mathrm{V}_{0}$ - volume de biogás corrigido $\left(\mathrm{m}^{3}\right)$;

$\mathrm{P}_{0}$ - pressão corrigida do biogás (101599,93 Pa);

$\mathrm{T}_{0}$ - temperatura corrigida do biogás $(293,15 \mathrm{~K})$;

$\mathrm{V}_{1}$ - volume do gás no gasômetro $\left(\mathrm{m}^{3}\right)$;

$\mathrm{P}_{1}$ - pressão do biogás no instante da leitura $(91239,99 \mathrm{~Pa})$;

$\mathrm{T}_{1}$ - temperatura do biogás, em $\mathrm{K}$, no instante da leitura.

As análises estatísticas consistiram de um estudo de regressão com os modelos não lineares sigmoide de Boltzmann, Gompertz e Logístico, cujas equações são apresentadas na Tabela 1. Os valores de produção de biogás estão apresentados por kg de substrato.

Tabela 1. Equações dos modelos não lineares ajustados à produção acumulada de biogás.

\begin{tabular}{lc}
\hline \multicolumn{1}{c}{ Modelo } & Equação \\
\hline Sigmoide de Boltzmann & $\mathrm{Y}_{\mathrm{i}}=\mathrm{AI}+(\mathrm{AS}-\mathrm{AI}) /(1+\exp ((\mathrm{PI}-\mathrm{x}) / \mathrm{Inc}))+\mathrm{u}_{\mathrm{i}}$ \\
Gompertz & $\mathrm{Y}_{i}=\mathrm{AS} .(\exp (-\exp (-$ Inc. $(\mathrm{x}-\mathrm{PI}))))+\mathrm{u}_{\mathrm{i}}$ \\
Logístico & $\mathrm{Y}_{i}=\mathrm{AS} /(1+\exp (-$ Inc. $(\mathrm{x}-\mathrm{PI})))+\mathrm{u}_{\mathrm{i}}$ \\
\hline
\end{tabular}

Fonte: Elaboração dos autores (2017).

em que:

$$
\begin{aligned}
& \mathrm{u}_{i}=\phi^{1} \mathrm{u}_{i}-1+\cdots+\phi_{p} \mathrm{u}_{i-\mathrm{p}}+\varepsilon_{i} \\
& i=1, \ldots, \mathrm{n}
\end{aligned}
$$

n o número de medições do biogás acumulado;

$\mathrm{u}_{i}$ é o resíduo do ajuste na i-ésima medição;

$\phi_{1}$ é o parâmetro autorregressivo de ordem 1 AR(1);

$\mathrm{u}_{i-1}$ é o resíduo do ajuste no tempo imediatamente anterior à i-ésima medição;

$\phi_{p}$ é o parâmetro autorregressivo de ordem $\mathrm{p}$;

$\mathrm{u}_{i}$ - p é o resíduo do ajuste em $\mathrm{p}$ tempos anterior à i-ésima medição;

$\varepsilon_{i}$ é o ruído branco com distribuição normal.

Nas equações apresentadas na Tabela $1, \mathrm{Y}_{i}$ é o volume de biogás acumulado na semana i, $A I=$ Assíntota Inferior, que indica o volume de biogás no momento da instalação, $A S=$ Assíntota Superior, refere-se ao volume máximo de biogás acumulado pelo sistema, $P I=$ Ponto de Inflexão, momento no qual ocorre a máxima liberação de biogás e Inc = Inclinação da curva no PI, indicando a máxima 
liberação de biogás, $x=$ a variável semana. Para a estrutura de erros independentes tem-se que os parâmetros são nulos, então $u_{i}=\varepsilon_{i}$ (PRADO et al., 2013b).

A normalidade dos resíduos foi avaliada por meio do teste de hipótese de Shapiro-Wilk (SHAPIRO; WILK, 1965), em que a hipótese nula é de que os resíduos seguem uma distribuição normal. Para a variância residual foi utilizado o teste de Breusch-Pagan (BREUSCH; PAGAN, 1979), cuja hipótese nula é de que os resíduos são homocedásticos e a hipótese de autocorrelação residual de primeira ordem foi avaliada pelo teste de Durbin-Watson (DURBIN; WATSON, 1950), a hipótese nula para este teste é a de independência dos resíduos.

A seleção do modelo mais adequado para explicar o acúmulo de Biogás nas semanas do estudo foi feita com base na precisão dos ajustes e obedeceu aos critérios que são mais utilizados na área para comparar modelos com diferentes números de parâmetros (FERNANDES et al., 2014; PRADO et al, 2013a): desvio-padrão residual (DPR), sendo mais eficiente o modelo que apresentar menor valor de DPR, critério de informação de Akaike (AIC), o modelo com menor valor de AIC é mais eficiente, e o coeficiente de determinação ajustado ( $\left.R^{2} a j\right)$, o modelo com maior valor para $R^{2}$ aj ajusta melhor os dados.

Os parâmetros dos modelos foram obtidos por meio da função "gnls" do software R (R DEVELOPMENT CORE TEAM, 2014), que usa o método de mínimos quadrados generalizados e o algoritmo de Gauss-Newton. Assim como a estimação dos parâmetros, as análises de resíduos, os avaliadores de qualidade e todos os gráficos também foram implementados no software R.

\section{Resultados e discussão}

Os resultados da análise dos resíduos (Tabela 2) são expressos pela significância dos testes de Shapiro-Wilk, Breusch-Pagan e Durbin-Watson. Conforme indicaram os resultados, os pressupostos de normalidade residual e de homogeneidade de variâncias se verificaram para todos os modelos ( $p$ $>0,05)$. No caso da independência dos resíduos, o teste de Durbin-Watson identificou a presença de autocorrelação para o modelo Gompertz nos dois tratamentos e para o modelo Logístico no tratamento $M+B$, portanto, na sequência dos resultados serão considerados os modelos com parâmetro $\mathrm{AR}(1)$ apenas quando significativos.

Tabela 2. Valores $p$ das estatísticas dos testes Shapiro-Wilk, Breusch-Pagan e Durbin-Watson dos resíduos dos modelos sigmoide de Boltzmann, Gompertz, Logístico para os tratamentos maravalha + bagaço de cana $(\mathrm{M}+\mathrm{B})$ e cama de maravalha (MAR).

\begin{tabular}{lcccccc}
\hline \multirow{2}{*}{ Modelo } & \multicolumn{3}{c}{ M+B } & \multicolumn{3}{c}{ MAR } \\
\cline { 2 - 7 } & $\begin{array}{c}\text { Shapiro- } \\
\text { Wilk }\end{array}$ & $\begin{array}{c}\text { Breusch- } \\
\text { Pagan }\end{array}$ & $\begin{array}{c}\text { Durbin- } \\
\text { Watson }\end{array}$ & $\begin{array}{c}\text { Shapiro- } \\
\text { Wilk }\end{array}$ & $\begin{array}{c}\text { Breusch- } \\
\text { Pagan }\end{array}$ & $\begin{array}{c}\text { Durbin- } \\
\text { Watson }\end{array}$ \\
\hline Sigmoide de Boltzmann & 0,17 & 0,26 & 0,31 & 0,31 & 0,36 & 0,14 \\
Gompertz & 0,54 & 0,36 & $<0,01$ & 0,19 & 0,21 & $<0,01$ \\
Logístico & 0,87 & 0,21 & 0,03 & 0,46 & 0,06 & 0,07 \\
\hline
\end{tabular}

Fonte: Elaboração dos autores (2017)

Esses resultados concordam com os estudos de Prado et al. (2013a) e Fernandes et al. (2014) que encontraram estruturas de erros dependentes em ajuste dos modelos Gompertz e Logístico a da- 
dos obtidos por meio de medidas ao longo do tempo. Para o modelo sigmoide de Boltzmann, os erros foram considerados independentes nos dois tratamentos.

As estimativas dos parâmetros dos modelos ajustados para ambos os tratamentos (Tabela 3) não são muito próximas, isto é, existem diferenças entre os modelos apresentados para um mesmo tratamento.

Tabela 3. Estimativas dos parâmetros com erro padrão para o ajuste do modelo sigmoide de Boltzmann, Gompertz e Logístico com os tratamentos maravalha + bagaço de cana ( $\mathrm{M}+\mathrm{B})$ e cama de maravalha (MAR).

\begin{tabular}{|c|c|c|c|c|c|c|}
\hline \multirow{2}{*}{ Tratamento } & \multirow{2}{*}{ Modelo } & \multicolumn{5}{|c|}{ Parâmetros } \\
\hline & & Al & AS & Inc & PI & $\phi$ \\
\hline \multirow{3}{*}{$M+B$} & $\begin{array}{l}\text { Sigmoide de } \\
\text { Boltzmann }\end{array}$ & $8,12 \pm 2,51$ & $129,53 \pm 2,44$ & $1,42 \pm 0,10$ & $4,95 \pm 0,10$ & \\
\hline & Logístico AR(1) & - & $133,88 \pm 3,79$ & $0,58 \pm 0,03$ & $4,82 \pm 0,16$ & $0,21 \pm 0,39$ \\
\hline & Gompertz AR(1) & - & $128,70 \pm 13,66$ & $0,45 \pm 0,07$ & $4,51 \pm 0,32$ & $0,94 \pm 0,10$ \\
\hline \multirow{3}{*}{ MAR } & $\begin{array}{l}\text { Sigmoide de } \\
\text { Boltzmann }\end{array}$ & $4,12 \pm 1,63$ & $43,62 \pm 1,06$ & $1,33 \pm 0,17$ & $4,21 \pm 0,17$ & \\
\hline & Logístico & - & $45,05 \pm 1,33$ & $0,58 \pm 0,04$ & $3,93 \pm 0,17$ & \\
\hline & Gompertz AR(1) & - & $49,35 \pm 4,01$ & $0,32 \pm 0,05$ & $3,09 \pm 0,37$ & $0,37 \pm 0,38$ \\
\hline
\end{tabular}

Fonte: Elaboração dos autores (2017)

O parâmetro AS é o valor de maior importância, pois se refere ao volume máximo de biogás produzido pelo sistema; por meio dele é que poderão ser dimensionados os biodigestores e estimada a quantidade de energia a ser produzida pelo sistema e o custo para a produção desta.

Para o tratamento $\mathrm{M}+\mathrm{B}$, os modelos Logístico, Gompertz e sigmoide de Boltzmann estimam aproximadamente os mesmos valores para o parâmetro AS, enquanto que para o tratamento MAR o maior valor de AS é estimado pelo modelo Gompertz AR(1).

Apenas o modelo sigmoide de Boltzmann apresenta um parâmetro para modelar o início da curva de acúmulo de biogás, Al, de menor interesse prático, a estimativa deste parâmetro representa a quantidade de biogás acumulada ao final da semana de instalação. Comparando os parâmetros Al do modelo sigmoide de Boltzmann ajustado ao tratamento $\mathrm{M}+\mathrm{B}$ com o ajustado ao tratamento MAR é possível verificar que já na semana de instalação, o tratamento $\mathrm{M}+\mathrm{B}$ produz em média o dobro de biogás do tratamento MAR. De acordo com Sousa (2014), a composição química essencialmente orgânica faz do bagaço de cana uma fonte potencial de geração de energia renovável.

Queiroz (2003) obteve bons resultados com o modelo Gompertz ao modelar a produção acumulada de biogás com tempos de retenção hidráulica de 84 e 77 dias, utilizando no seu experimento dejetos sólidos de bovinos destinados à produção de leite, quando o comparou com o modelo exponencial, sem considerar a correlação residual. Lopes (2007) também obteve bons resultados com o modelo Gompertz na modelagem da produção acumulada de biogás em rede de biodigestores anaeróbios.

O parâmetro PI estimado pelos modelos sigmoide de Boltzmann e Logístico serve para indicar o momento no qual ocorre $50 \%$ do total da produção de biogás pelo biodigestor; no modelo Gompertz, ele estima cerca de $37 \%$ deste total produzido. Antes do PI, observa-se um aumento na produção do biogás (concavidade voltada para cima), no PI ocorre a taxa máxima de liberação de biogás e após o PI começa a decair a produção do biogás (concavidade voltada para baixo). 
Para o tratamento $\mathrm{M}+\mathrm{B}$ o PI foi estimado próximo ao final da $5^{\mathrm{a}}$ semana pelos três modelos, ao passo que, para o tratamento MAR, o PI foi estimado em momentos diferentes pelos modelos.

O parâmetro Inc se refere ao aumento da velocidade de acúmulo de biogás no ponto de inflexão, no modelo sigmoide de Boltzmann este parâmetro foi cerca de três vezes maior se comparado aos demais modelos.

De modo geral, todos os modelos apresentaram ajustes satisfatórios nos dois tratamentos (Tabela 4) e o modelo não linear sigmoide de Boltzmann apresentou os menores valores para o DPR e AIC e maior valor no $\mathrm{R}^{2}$ aj tanto para o tratamento $\mathrm{M}+\mathrm{B}$ quanto para MAR, sendo, portanto, o mais adequado para descrever a produção de biogás de resíduo proveniente de camas de suínos compostas de $\mathrm{M}+\mathrm{B}$ ou somente em MAR em biodigestores do tipo batelada.

Tabela 4. Estimativas dos critérios de seleção: coeficiente de determinação ajustado $\left(R^{2} a j\right)$, desvio-padrão residual (DPR) e critério de Informação de Akaike (AIC), para os modelos ajustados, na descrição do volume de biogás produzido pelos tratamentos maravalha + bagaço de cana $(\mathrm{M}+\mathrm{B})$ e cama de maravalha (MAR).

\begin{tabular}{ccccc}
\hline \multirow{2}{*}{ Tratamento } & Modelo & \multicolumn{2}{c}{ Critérios de Seleção } \\
\cline { 2 - 5 } & Sigmoide de Boltzmann & 0,9957 & 2,22 & 53,82 \\
\multirow{2}{*}{ M+B } & Gompertz AR(1) & 0,9025 & 12,6 & 70,93 \\
& Logístico AR(1) & 0,9945 & 2,94 & 60,96 \\
\hline \multirow{2}{*}{ MAR } & Sigmoide de Boltzmann & 0,9927 & 1,26 & 41,4 \\
& Gompertz AR(1) & 0,9812 & 2,05 & 52,09 \\
& Logístico & 0,9909 & 1,44 & 43,79 \\
\hline
\end{tabular}

Fonte: Elaboração dos autores (2017).

Esses resultados concordam com Florentino, Biscaro e Passos (2010) que compararam diversos modelos sigmoidais aplicados na determinação da atividade metanogênica específica, por meio da soma de quadrados dos resíduos, chegando a conclusão de que o modelo sigmoide de Boltzmann é superior a todos os outros modelos sigmoidais.

O gráfico que ilustra o ajuste do modelo sigmoide de Boltzmann à produção acumulada de biogás ao longo das semanas pode ser observado na Figura 2. 
Figura 2. Curva ajustada do modelo sigmoide de Boltzmann ao volume de biogás acumulado para os tratamentos maravalha + bagaço de cana $(M+B)$ e cama de maravalha (MAR) com intervalos de confiança.

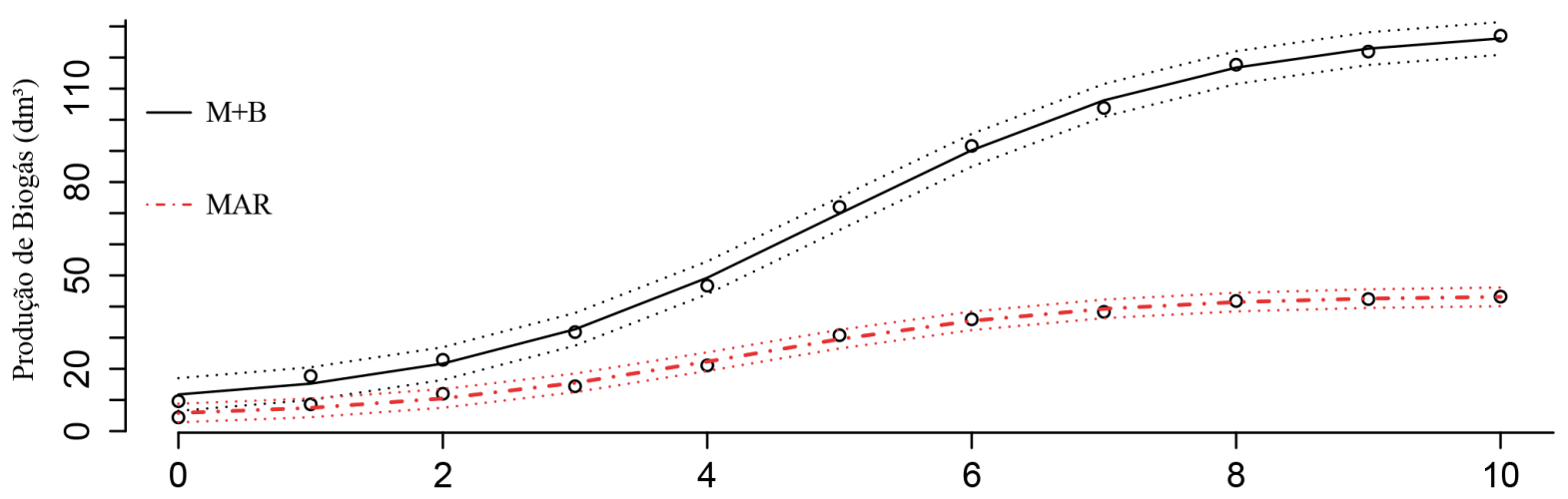

Fonte: Elaboração dos autores (2017).

O distanciamento das curvas para os dois tratamentos evidencia a superioridade da cama composta de $\mathrm{M}+\mathrm{B}$ para a produção de biogás, indicando que, se disponível, o bagaço de cana chega a triplicar a produção de biogás do que se usado somente MAR. É possível notar também que praticamente não há interseção dos intervalos de confiança a partir da primeira semana.

$\mathrm{O}$ ajuste do modelo Gompertz, modelo que apresentou a pior qualidade de ajuste aos dados de produção acumulada de biogás, quando comparado com os outros dois modelos, está ilustrado na Figura 3.

Figura 3. Curva ajustada do modelo Gompertz ao volume de biogás acumulado para os tratamentos de maravalha + bagaço de cana $(M+B)$ e cama de maravalha (MAR) com intervalos de confiança.

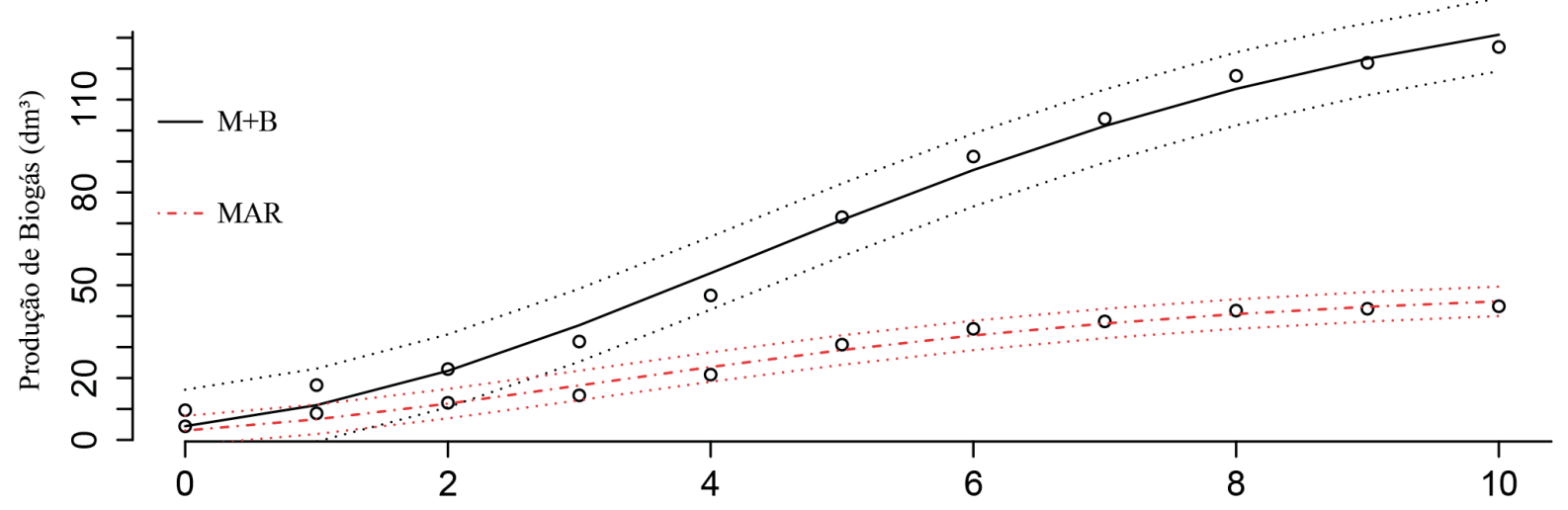

Fonte: Elaboração dos autores (2017).

A pior qualidade de ajuste do modelo Gompertz evidencia que a produção acumulada de biogás é um processo simétrico. Lopes (2007) e Queiroz (2003) obtiveram melhores ajustes para o modelo Gompertz, todavia o compararam com o exponencial, o qual não possui caráter sigmoidal. 
A Figura 4 ilustra o ajuste do modelo Logístico à produção acumulada de biogás. Este modelo se aproximou mais do modelo sigmoide de Boltzmann em qualidade de ajuste.

Figura 4. Curva ajustada do modelo Logístico ajustada ao volume de biogás acumulado para os tratamentos de maravalha + bagaço de cana $(M+B)$ e cama de maravalha (MAR) com intervalos de confiança.

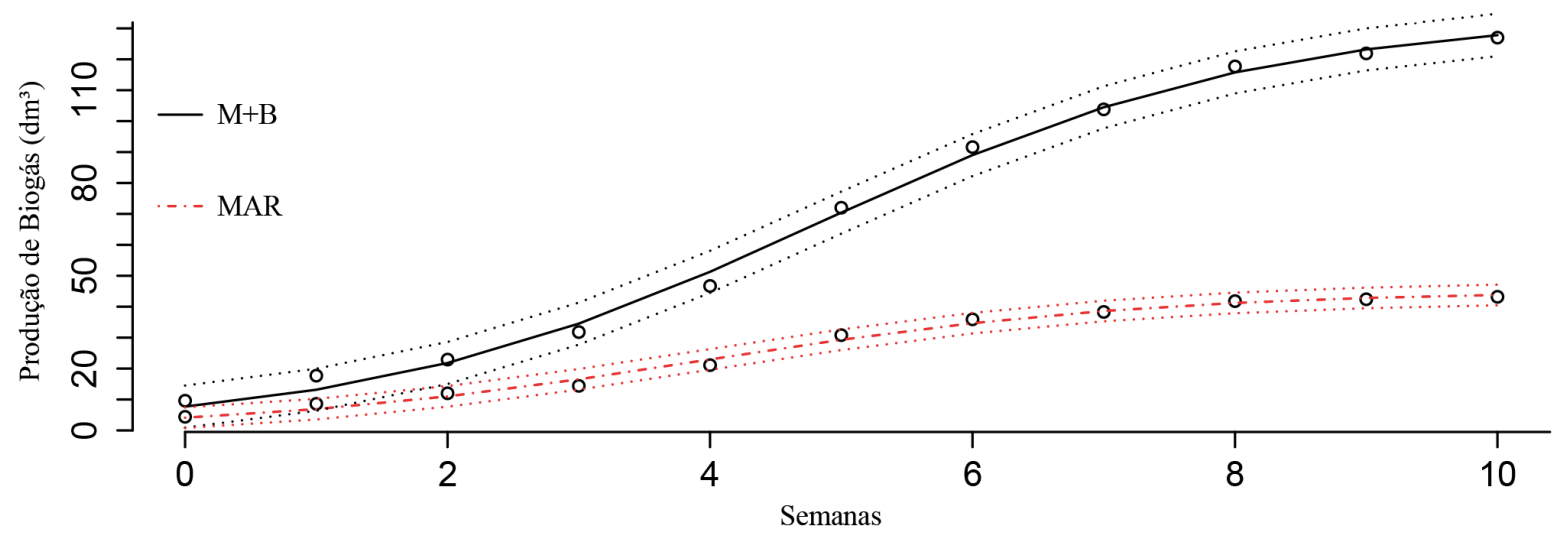

Fonte: Elaboração dos autores (2017).

Os resultados obtidos mostram de maneira geral que diferentes composições dos dejetos utilizados nos biodigestores levam a diferentes produções de biogás. Costa (2009), Miranda et al. (2012), Aires et al. (2009) e Orrico Júnior, Orrico e Lucas Júnior (2009) também obtiveram diferentes produções de biogás ao utilizar diferentes composições nos biodigestores. A curva de produção acumulada de biogás apresenta caráter sigmoidal, fato observado também por Florentino, Biscaro e Passos (2010), com fortes evidências de ser simétrica em relação ao ponto de inflexão.

É possível observar uma similaridade de produção entre os dois tratamentos no decorrer das semanas avaliadas, indicando que as curvas de produção de biogás oriundas de diferentes materiais de cama sobreposta de suínos seguem o mesmo padrão.

Para dimensionar a produção de biogás deve-se considerar o ajuste pelo modelo sigmoide de Boltzmann, o qual indica serem necessários cerca de $8 \mathrm{~kg}$ de cama composta de $\mathrm{M}+\mathrm{B}$ para produzir $1 \mathrm{~m}^{3}$ de biogás enquanto para cama de MAR são necessários cerca de $23 \mathrm{~kg}$ para produzir $1 \mathrm{~m}^{3}$ de biogás. Resultados semelhantes foram obtidos por Costa (2009) ao utilizar cama de frango com serra de pinus e por Fukayama (2008) ao utilizar cama de frango.

\section{Conclusões}

O modelo sigmoide de Boltzmann é superior aos demais modelos para descrever o volume de biogás acumulado tanto para cama composta de maravalha + bagaço de cana quanto para maravaIha em biodigestor do tipo batelada com tempo de retenção hidráulica de 60 dias.

Para o dimensionamento da produção de energia por meio de biogás é mais eficiente utilizar o modelo sigmoide de Boltzmann.

O tratamento maravalha + bagaço de cana produz, em média, um volume de biogás três vezes maior que o tratamento maravalha. 


\title{
Agradecimentos
}

Ao CNPq pela bolsa de estudos.

\section{Non-linear models adjusted to the accumulated production of biogas originating from swine deep bedding}

\begin{abstract}
There are currently many issues concerning the shortage of rainfall, one being the low production of hydroelectric power and the consequent rise in electricity rates. Farmers are directly affected, since such variation causes higher production costs. One way out for farmers is to use the waste derived from agricultural and livestock activities to produce biogas with biodigesters, and generate power. In addition to the benefit of biogas and energy, the use of residue from biodigesters promotes rural sanitation and produces a high quality biofertilizer. This work is a regression study with the Boltzmann sigmoid, the Gompertz and the Logistic non-linear models using data from a project developed by Sousa (2014), with the objective of verifying which non-linear regression model better fits the accumulated production of biogas from batch biodigesters, with diluted material from swine deep bedding prepared with wood shavings and sugarcane bagasse, and only with wood shavings. It was verified that the Boltzmann sigmoid non-linear model is superior to the other models and, consequently, more efficient for measuring the production of biogas and electric power.
\end{abstract}

Keywords: Electric power. Biodigesters. Boltzmann sigmoid.

\section{Referências}

AIRES, A. M.; LUCAS JUNIOR, J.; FUKAYAMA, E. H.; MACHADO, C. R.; GUIDOLIM, D. G. F. Biodigestão anaeróbia da cama de frangos de corte com ou sem separação das frações sólida e líquida sobre a produção de biogás e a qualidade do biofertilizante. In: CONGRESSO DE MEIO AMBIENTE DA AUGM, 6, 2009, São Carlos. Anais... São Carlos: UFSCar, 2009. p.1-15. Disponível em: <http:// www.ambiente-augm.ufscar.br/uploads/A2-120.pdf >. Acesso em: 25 fev. 2016.

AVACI, A. B.; SOUZA, S. M. de; CHAVES, L. I.; NOGUEIRA, C. E. C.; NIEDZIALKOSKI, R. K.; SECCO, D. Avaliação econômico-financeira da microgeração de energia elétrica proveniente de biogás da suinocultura. Revista Brasileira de Engenharia Agrícola e Ambiental, Campina Grande, v.17, n.4, p.456-462, abr. 2013. Disponível em: <http://www.scielo.br/scielo.php?script=sci_arttext\&pi$\mathrm{d}=\mathrm{S} 1415-43662013000400015 \& \mathrm{lng}=\mathrm{en} \& \mathrm{nrm}=$ iso $>$. Acesso em: 06 fev. 2016.

BREUSCH, T. S.; PAGAN, A. R. A Simple test for heteroscedasticity and random coefficient variation. Econometrica, New York, v.47, n.5, p.1287-1294, sep. 1979. Disponível em: <https://www.aae. wisc.edu/aae637/handouts/breusch_pagan_hetero_test_article.pdf $>$. Acesso em: 02 dez. 2015.

CAETANO, L. Proposição de um sistema modificado para quantificação de biogás. 1985. $75 f$. Dissertação (Mestrado em Energia na Agricultura) - Faculdade de Ciências Agrárias, Universidade Estadual Paulista, Botucatu, 1985. 
CARNEIRO, A. P. S.; MUNIZ, J. A.; CARNEIRO, P. L. S.; MALHADO, C. H. M.; MARTINS FILHO, R.; SILVA, F. F. e. Identidade de modelos não lineares para comparar curvas de crescimento de bovinos da raça Tabapuã. Pesquisa Agropecuária Brasileira, Brasília, v.49, p. 57-62, jan. 2014. Disponível em: <https://seer.sct.embrapa.br/index.php/pab/article/view/18183>. Acesso em: 04 fev. 2016.

COLDEBELLA, A.; SOUZA, S. N. M. de; FERRI, P.; KOLLING, E. M. Viabilidade da geração de energia elétrica através de um motor gerador utilizando biogás da suinocultura. Informe GEPEC, Toledo, v.12, n.2, p.44-55, 2008. Disponível em: <http://e-revista.unioeste.br/index.php/gepec/article/ view/2186/1698>. Acesso em: 15 mar. 2016

CORRÊA FILHO, L. C.; ANDRADE, E. T. de; MARTINAZZO, A. P.; D'ANDREA, E. M.; SOUSA, F. A. de; FIGUEIRA, V. G. Cinética de secagem, contração volumétrica e análise da difusão líquida do figo (Ficus carica L.). Revista Brasileira de Engenharia Agrícola e Ambiental, Campina Grande, v.19, n.8. p.797-802, 2015. Disponível em: <http://www.scielo.br/pdf/rbeaa/v19n8/1807-1929-rbeaa-19-08-0797>. Acesso em: 10 fev. 2016.

COSTA, L. V. C. Biodigestão anaeróbia da cama de frango associada ou não ao biofertilizante obtido com dejetos de suínos: produção de biogás e qualidade do biofertilizante. 2009. 89f. Dissertação (Mestrado em Produção Animal) - Faculdade de Ciências Agrárias e Veterinárias, Universidade Estadual Paulista, Jaboticabal, 2009.

DRAPER, N. R.; SMITH, H. Applied regression analysis. 3. ed. New York: J. Wiley, 1998.

DURBIN, J.; WATSON G. S. Testing for serial correlation in least squares regression I. Biometrika, London, v.37, n.3/4, p.409-428, dec. 1950. Disponível em: <https://www.jstor.org/stable/2332391>. Acesso em: 15 mar. 2016

FLORENTINO, H. O.; BISCARO, A. F. V.; PASSOS, J. R. S. Funções sigmoidais aplicadas na determinação da atividade metanogênica específica - AME. Revista Brasileira de Biometria, São Paulo, v.28, n.1, p.141-150, mar. 2010. Disponível em: <http://jaguar.fcav.unesp.br/RME/fasciculos/v28/ v28_n1/A10_Helenice.pdf >. Acesso em: 10 fev. 2016.

FUKAYAMA, E. H. Características quantitativas e qualitativas da cama de frango sob diferentes reutilizações: efeitos na produção de biogás e biofertilizante. 2008. 96f. Tese (Doutorado em Produção Animal) - Faculdade de Ciências Agrárias e Veterinárias, Universidade Estadual Paulista, Jaboticabal, 2008.

FERNANDES, T. J.; PEREIRA, A. A.; MUNIZ, J. A.; SAVIAN, T. V. Seleção de modelos não lineares para a descrição das curvas de crescimento do fruto do cafeeiro. Revista Coffee Science, Lavras, v.9, n.2, p.207-215, jun. 2014. Disponível em: <http://www.coffeescience.ufla.br/index.php/Coffeescience/article/view/618/pdf_86>. Acesso em: 15 mar. 2016.

LOPES, G. N. Modelagem não linear da produção acumulada de biogás em rede de biodigestores anaeróbios. Revista Agro@mbiente on line, Boa Vista, v.1, n.1, p.37-40, jul./dez. 2007. Disponível em: <http://revista.ufrr.br/agroambiente/article/view/144/80>. Acesso em: 10 fev. 2016.

LUNA, M. L. D. de; LEITE, V. D.; LOPES, W. S.; SOUSA, J. T. de; SILVA, S. A. Tratamento anaeróbio de resíduos orgânicos com baixa concentração de sólidos. Engenharia Agrícola, Jaboticabal, v.29, n.1, p.113-121, mar. 2009. Disponível em: <http://www.scielo.br/scielo.php?script=sci_arttext\&pid=S0100-69162009000100012\&lng=pt\&nrm=iso >. Acesso em: 05 ago. 2017. 
MARTINS, F. M.; OLIVEIRA, P. A. V. de. Análise econômica da geração de energia elétrica a partir do biogás na suinocultura. Engenharia Agrícola, Jaboticabal, v. 31, n. 3, p. 477 486, jun. 2011. Disponível em: <http://www.scielo.br/scielo.php?script=sci_arttext\&pi$\mathrm{d}=$ S0100-69162011000300008\&Ing=pt\&nrm=iso >. Acesso em: 05 ago. 2017.

MIRANDA, A. P.; LUCAS JUNIOR, J. de; THOMAZ, M. C.; PEREIRA, G. T.; FUKAYAMA, E. H. Anaerobic biodigestion of pigs feces in the initial, growing and finishing stages fed with diets formulated with corn or sorghum. Engenharia Agrícola, Jaboticabal, v.32, n.1, fev. 2012. Disponível em: <http:// www.scielo.br/scielo.php?script=sci_arttext\&pid =S0100-69162012000100006\&lng =en\&nrm $=\mathrm{i}-$ so >. Acesso em: 02 mar. 2017.

NISHIMURA, R. Análise de balanço energético de sistema de produção de biogás em granja de suínos: implementação de aplicativo computacional.2009. 84f. Dissertação (Mestrado em Engenharia Elétrica) - Universidade Federal de Mato Grosso do Sul, Campo Grande, 2009.

ORRICO JÚNIOR, M. A. P.; ORRICO A. C. A. LUCAS JÚNIOR, J. DE. Biodigestão anaeróbia de dejetos de suínos com e sem separação da fração sólida em diferentes tempos de retenção hidráulica. Engenharia Agrícola, Jaboticabal, v.29, n.3, p.474-482, set. 2009. Disponível em: <http://www.scielo. br/scielo.php?script=sci_arttext\&pid=S0100-69162009000300014\&lng $=$ en\&nrm $=i$ iso $>$. Acesso em: 01 fev. 2016.

PRADO, T. K. L. do; SAVIAN, T. V.; MUNIZ, J. A. Ajuste dos modelos Gompertz e Logístico aos dados de crescimento de frutos de coqueiro anão verde. Ciência Rural, Santa Maria, v.43, n.5, p.803-809, maio, 2013a. Disponível em: <http://www.scielo.br/scielo.php?script=sci_arttext\&pi$\mathrm{d}=$ S0103-84782013000500008\&lng=en\&nrm=iso $>$. Acesso em: 16 fev. 2016.

PRADO, T. K. L. do; MUNIZ, J.A.; SAVIAN, T.V.; SÁFADI, T. Ajuste do modelo Logístico na descrição do crescimento de frutos de coqueiro anão por meio de algoritmos iterativos. Revista Brasileira de Biometria, São Paulo, v.31, n.2, p.216-232, 2013b. Disponível em: <http://jaguar.fcav.unesp.br/ RME/fasciculos/v31/v31_n2/A4_Thalita_Joel.pdf>. Acesso em: 15 dez. 2016

QUEIROZ, S.C. Modelagem da produção acumulada de biogás em biodigestores tipo batelada segundo a porcentagem de inóculo adicionado utilizando os modelos de regressão não-linear de Gompertz e exponencial. 2003. 112f. Tese (Doutorado em Energia na Agricultura) - Faculdade de Ciências Agrárias, Universidade Estadual Paulista, Botucatu, 2003.

R DEVELOPMENT CORE TEAM. R: a language and environment for statistical computing. Vienna: $R$ Foundation for Statistical Computing, 2014. Disponível em: <https://www.R-project.org>. Acesso em: 21 nov. 2014.

SHAPIRO, S. S.; WILK, M. B. An analysis of variance test for normality. Biometrika, London, v.52, n.3/4, p.591-611, dec. 1965. Disponível em: <https://www.jstor.org/stable/2333709>. Acesso em: 18 fev. 2016.

SOUSA, F. A. Análise de fatores ambientais na criação de suínos em camas sobrepostas e produção de biogás. 2014. 125f. Tese (Doutorado em Engenharia Agrícola) - Universidade Federal de Lavras, Lavras, 2014. 
SOUZA, I. F.; KUNZLE NETO, J. E.; MUNIZ, J. A.; GUIMARÃES, R. M.; SAVIAN, T. V.; MUNIZ, F. R. Fitting nonlinear autoregressive models to describe coffee seed germinations. Ciência Rural, Santa Maria, v.44, n.11, p.2016-2021, nov. 2014. Disponível em: <http://www.scielo.br/scielo.php?s$\mathrm{cript}=$ sci_arttext\&pid=S0103-84782014001102016\&lng=en\&nrm=iso>. Acesso em: 02 nov. 2016.

\section{Histórico editorial:}

Submetido em: 11/02/2017

Aceito em: 08/08/2017

\section{Como citar:}

ABNT

SILVEIRA, S. C.; MUNIZ, J. A.; SOUSA, F. A.; CAMPOS, A. T. Modelos não lineares ajustados à produção acumulada de biogás provenientes de camas sobrepostas de suínos. Revista Agrogeoambiental, Pouso Alegre, v. 10, n. 3, p. 91-103, jul./set. DOI: http://dx.doi.org/10.18406/2316-1817v10n320181168

APA

SILVEIRA, S. C., MUNIZ, J. A., SOUSA, F. A. \& CAMPOS, A. T. (2018). Modelos não lineares ajustados à produção acumulada de biogás provenientes de camas sobrepostas de suínos. Revista Agrogeoambiental, 10 (3), 91-103. DOI: http://dx.doi.org/10.18406/2316-1817v10n320181168

$\underline{\text { ISO }}$

SILVEIRA, S. C.; MUNIZ, J. A.; SOUSA, F. A. e CAMPOS, A. T. Modelos não lineares ajustados à produção acumulada de biogás provenientes de camas sobrepostas de suínos. Revista Agrogeoambiental, 2018 , vol. 10, n. 3, pp. 91-103. Eissn 2316-1817. DOI: http://dx.doi.org/10.18406/2316-1817v10n320181168

\section{VANCOUVER}

Silveira SC, Muniz JA, Sousa FA, Campos, AT. Modelos não lineares ajustados à produção acumulada de biogás provenientes de camas sobrepostas de suínos. Rev agrogeoambiental. 2018. jul./set.; 10(3): 91-103. DOI: http://dx.doi.org/10.18406/2316-1817v10n320181168 


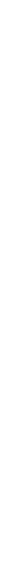

